\title{
The Effect of Self-Driving Car on Urban Traffic
}

\author{
Zaiqiang Ku, Ting Liao* \\ College of Mathematics and Physics, Huanggang Normal University, Hubei, China \\ Email: *kzqhgnu@163.com
}

How to cite this paper: $\mathrm{Ku}, \mathrm{Z} . \mathrm{Q}$. and Liao, T. (2017) The Effect of Self-Driving Car on Urban Traffic. American Journal of Computational Mathematics, 7, 149-156. https://doi.org/10.4236/ajcm.2017.72013

Received: March 20, 2017

Accepted: June 11, 2017

Published: June 14, 2017

Copyright (C) 2017 by authors and Scientific Research Publishing Inc. This work is licensed under the Creative Commons Attribution International License (CC BY 4.0).

http://creativecommons.org/licenses/by/4.0/

\begin{abstract}
Based on the idea of infinitesimal analysis, we establish the basic model of relation between speed and flow. Since putting a certain amount of self-driving car will affect the average speed of mixed traffic flow, we choose the proportion of self-driving car to be a variable, denoted by $\mathrm{k}$. Based on the least square method, we find two critical values of $\mathrm{k}$ that are $38.63 \%$ and $68.26 \%$. When $\mathrm{k}$ $<38.63 \%$, the self-driving cars have a negative influence to the traffic. When $38.63 \%<\mathrm{k}<68.26 \%$, they have a positive influence to the traffic. When $\mathrm{k}>$ $68.26 \%$, they have significant improvement to the traffic capacity of the road.
\end{abstract}

\section{Keywords}

Self-Driving Car, Least Square Method, Mixing Speed, Traffic Flow

\section{Introduction}

In order to indicate the origin of self-driving, cooperating cars, the following background is worth mentioning.

\subsection{Background}

As we all known that the traffic capacity is limited in many areas of the United States owing to the number of lanes of roads. However, self-driving, cooperating cars have been provided a solution to increase the capacity of highways without increasing the number of lanes of roads. The self-driving, cooperating cars are popular with its advantages, for example, faster speed, faster acceleration, and shorter baking distance.

\subsection{Literature Review}

$\mathrm{Lu}$ [1] analyzed the influence of mixed traffic flow car's speed under different traffic flow on the highway. He studied the relationship between traffic flow and traffic speed change and built the model of speed-flow. At the same time, he also analyzed the impact of medium-sized car under different ratios on highway 
mixed traffic flow speed, researched the relation between the ratio of large and medium-sized cars with the changes of the rate of traffic and built relationship model between speed with the speed-large and the proportion of medium-sized cars. Daganzo and Knoop [2] derived the analytic form of the capacity formula inspired by analytic upper and lower bounds derived with variational theory for a version of the problem where cars are treated as a fluid. Daganzo and Lehe [3] considered a signalized street of uniform width and blocks of various lengths. It has shown that the macroscopic fundamental diagram of an arterial street controlled by traffic signals was defined by the solution of a linear program. Sorigueraa et al. [4] examined both the macroscopic and microscopic effects of different speed limits on a traffic stream. Jamshidnejad et al. [5] proposed a framework to interface and integrate macroscopic flow models and microscopic emission models. Yang et al. [6] addressed the planning and optimization of intermodal hub-and-spoke (IH \& S) network considering mixed uncertainties in both transportation cost and travel time. Celikoglu and Silgu [7] evaluated the performance of a dynamic approach to classify flow patterns reconstructed by a switching-mode macroscopic flow model considering a multivariate clustering method. Yang et al. [8] proposed a bi-objective hub-and-spoke (H \& S) network design problem with type-2 (T2) fuzzy transportation cost and travel time described by parametric secondary possibility distributions. Those give us some inspiration about research the mixed ratio of self-driving cars and the mixed speed of flows. We will consider mainly the delay time to self-driving cars deal with signal which results to the changes of the traffic flows.

\subsection{Our Approach}

We use the infinitesimal analysis method to establish the model of relationship between flow and velocity, and combine with the least square method to calculate driving the delivery ratio as a function of the average speed of mixed traffic flow. Using least square method can easily find the function relationship between the variables, and make the error between the predicted value and the actual value of sum of squares to a minimum. Therefore, it is reasonable for us to find the best function relation between the variables with the least squares approximation.

The rest of this paper is organized as follows. In Section 2, we describe the problem in detail. In Section 3, we establish the model and derive the solution based on the least square method. Finally, some conclusions are made in Section 4.

\section{Problem Description}

Traffic capacity is limited the designed capacity of the road networks. Once over the capacity of the road networks, drivers would experience long delays during peak traffic hours. Self-driving, cooperating cars have been proposed as a solution to increase capacity of highway without increasing number of lanes. Our model is required to solve the influence of traffic capacity about traffic lane 
number, peak or average, self-drive and percentage of self-driving. Further, to achieve a certain number, the number of self-drive capacity has a significant effect for roads, whether should set lanes for self-drive and corresponding policy change. By analysis, we proposed to decompose the problem into three subproblems:

1) Sure the driving cars with the self-drive cars in different sections of mixed traffic flow speed.

2) Built a model to deal with the impact of traffic lane number, peak or average model analysis, the driving and percentage of self-drive influence on traffic capacity.

3) Reaching a certain number, the number of self-drive consideration for self-drive lanes and pipe policy tendency, and find out under different number of lanes, the number of lanes and self-drive best delivery ratio.

\section{The Model}

\subsection{Definitions and Symbols}

In order to model the above mentioned problem, the following notations are employed (Table 1).

Since they drive on the proportion of directly affects the road traffic capacity, this article defined equilibrium and tipping point as follows.

Table 1. The list of notations.

\begin{tabular}{|c|c|c|}
\hline Symbol & Definition & Units \\
\hline $\bar{v}$ & The average velocity of mixed traffic flow & mile/hour \\
\hline$S_{\text {sum }}$ & The vehicle by the total distance of a road & mile \\
\hline$t_{\text {sum }}$ & Some sections of the vehicle by the total time & hour \\
\hline$l_{1}$ & The safe distance from driving & mile \\
\hline$l_{2}$ & The length of self-driving, cooperating cars & mile \\
\hline$k$ & The proportion of self-driving, cooperating cars & mile \\
\hline$s$ & Through a section of distance & mile \\
\hline$T_{1}$ & Through a road the total time of driving cars & hour \\
\hline$T_{2}$ & Through a road the total time of self-driving cars & hour \\
\hline$T_{3}$ & Under the influence of the driving cars, the self-drive delay time & hour \\
\hline$k$ & The proportion of self-driving & \\
\hline$Q$ & A road traffic flow & hour \\
\hline$\rho$ & Some sections of the vehicle density & \\
\hline$k_{j}$ & A road block coefficient & \\
\hline$v_{1}$ & The average speed of driving cars & mile/hour \\
\hline$v_{2}$ & The average speed of self-driving cars & mile/hour \\
\hline$v_{0}$ & The average speed of zero flow & mile/hour \\
\hline
\end{tabular}


Tipping point: The proportion of the self-driving, cooperating cars when selfdriving cars on road traffic capacity has the largest inhibition effect (Chen et al. [9]).

Equilibrium: The proportion of the self-driving, cooperating cars when selfdriving cars and driving cars and driving cars go to the Nash equilibrium point (Chen et al. [10]).

\subsection{Assumptions}

In order to establish the model, we make the following assumptions, which have been used extensively in Gupta and Dhiman [11], Qu et al. [12], and Tang et al [13].

Assumption 1. Self-driving cars evenly distributed in the driving cars.

Assumption 2. All the driving and the self-drive conductor are equal.

Assumption 3. Ignore to slope impact on the traffic of flow.

\subsection{The Foundation of Model}

We divide the establishing process of the model into the following two steps.

\section{Step 1 The mixed speed of self-driving cars and driving cars}

Based on our assumption that all the self-drive uniform distribution in the self-drive, as shown in Figure 1. In order to calculate the average speed of mixed traffic flow, we since the driving ratio of $10 \%$ (Chen et al. [14]).

$$
\begin{gathered}
\bar{v}=\frac{s_{\text {sum }}}{t_{\text {sum }}} \\
s_{\text {sum }}=\sum_{i=0}^{9}\left(\int_{t_{i-1}}^{t_{i}} \frac{\mathrm{d} s_{i}}{\mathrm{~d} t}+i\left(l_{1}+l_{2}\right)\right) \\
t_{\text {sum }}=T_{1}+T_{2}+k^{*} T_{3} \\
T_{1}=\frac{\sum_{i=0}^{8}\left(\int_{t_{i-1}}^{t_{i}} \frac{\mathrm{d} s_{i}}{\mathrm{~d} t}+i\left(l_{1}+l_{2}\right)\right)}{v_{1}} \\
T_{2}=\frac{s+9\left(l_{1}+l_{2}\right)}{v_{2}}
\end{gathered}
$$

According to (1)-(5), we can get the average speed of mixed traffic flow as

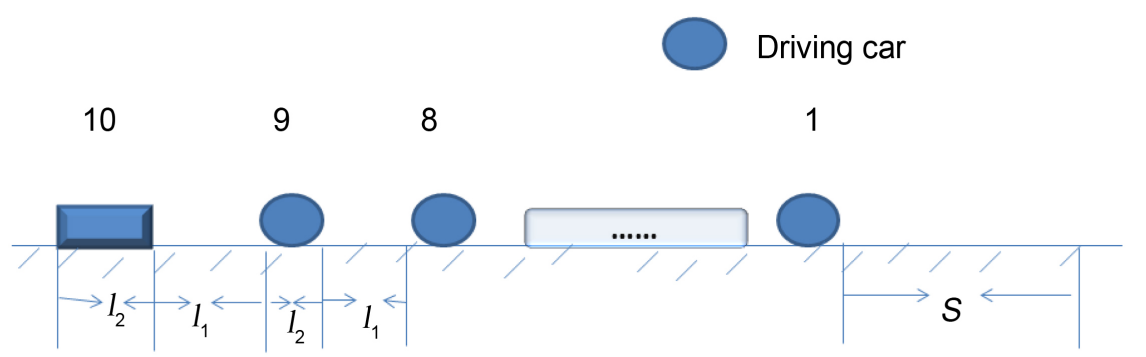

Figure 1. Hybrid vehicle distribution. 
follows.

$$
\bar{v}=\frac{\sum_{i=0}^{9}\left(\int_{t_{i-1}}^{t_{i}} \frac{\mathrm{ds} s_{i}}{\mathrm{~d} t}+i\left(l_{1}+l_{2}\right)\right)}{\frac{9 s+36\left(l_{1}+l_{2}\right)}{v_{1}}+\frac{s+9\left(l_{1}+l_{2}\right)}{v_{2}}+k^{*} T_{3}}
$$

\section{Step 2 Speed-flow model}

In the continuous traffic flow, average speed, the density of flow and between the following relations:

$$
Q=\rho^{*} \bar{v}
$$

We based on the hypothesis that density has a linear relation with the flow, can derive the average speed and flow rate of the quadratic parabola relationship model:

$$
Q=k_{j}\left(\bar{v}-\frac{\bar{v}^{2}}{v_{0}}\right)
$$

Among from them, the for block coefficient, the average of speed at the time of zero flow. In theory, when the traffic flow to maximum, the average speed of traffic flow half of vitamin to zero flow speed, while the maximum flow is traffic capacity.

\subsection{Solution and Result}

\section{Step 1 Establish automatic driving vehicle delay time}

Because of self-drive obstacle information processing, with the percentage of self-drive on different, delay would happen. According to the driving on the proportion of $50 \%$ had the greatest influence of traffic capacity [5] [15] and Arizona from driving the test data [4] [16] [17], on the proportion of self-drive delay time to simulate, you can get different delivery delay time under the proportion, as shown in Table 2. The computational experiments are carried out on a personal computer (Dell with Intel (R) Core (TM)i5-2450M CPU 2.50 GHZ and RAM $2.50 \mathrm{~GB}$ ), using the Microsoft Windows 7 operating system (Cheng et al. [18]).

\section{Step 2 The driving with the self-drive mixed average speed}

A safe distance from $l_{1}=0.06$ miles, conductor $l_{2}=0.0031$ miles, the distance by vitamin $s=200$ miles, the self-drive miles per hour, the speed of the driving miles per hour, the speed of the delay time of self-drive, in (6) available in the average speed of mixed traffic flow under different delivery ratio, the results are shown in Table 3.

According to the data in Table 3 , in order to establish the average velocity $\bar{v}$ and put in the function $\bar{v}(k)$.

Table 2. The delay time under different delivery ratios.

\begin{tabular}{ccccccccccc}
\hline Proportion & $10 \%$ & $20 \%$ & $30 \%$ & $40 \%$ & $50 \%$ & $60 \%$ & $70 \%$ & $80 \%$ & $90 \%$ & $100 \%$ \\
\hline$t_{3}(s)$ & 0.3 & 0.8 & 1.2 & 1.6 & 1.85 & 1.6 & 1.2 & 0.9 & 0.6 & 0.1
\end{tabular}


Table 3. Average velocity under different delivery ratios.

\begin{tabular}{ccccccccccc}
\hline Proportion & $10 \%$ & $20 \%$ & $30 \%$ & $40 \%$ & $50 \%$ & $60 \%$ & $70 \%$ & $80 \%$ & $90 \%$ & $100 \%$ \\
\hline $\bar{v}$ & 39.62 & 38.47 & 37.75 & 37.05 & 36.88 & 38.47 & 40.83 & 43.02 & 45.46 & 49.38 \\
\hline
\end{tabular}

Using the MATLAB software, first of all, the relationship between the scatterplot, found that increasing its change trend to decline after the first, in line with the basic facts.

Next, by adopting the idea of least square method, average speed can be on different on the proportion of mixed traffic flow with the quadratic curve fitting function of $\bar{v}(k)=a k^{2}+b k+c$, the average speed of mixed traffic flow and the function relation between driving the proportion is the function:

$$
\bar{v}=0.3259 k^{2}-2.5180 k+41.9942
$$

When the driving on the tipping point $k_{1}=38.63 \%$, at this point the drive to prevent traffic capacity of the largest. When driving on the proportion of equilibrium $k_{2}=68.26 \%$, the point where the driving influence on traffic capacity. When the delivery ratio $k<k_{1}$, self-drive for road traffic capacity has obvious inhibiting effect. When put in the proportion of $k_{1}<k<k_{2}$, self-drive for road traffic capacity and some improvement. When dropping ratio $k_{1}>k_{2}$, consider setting up self-drive lanes.

\section{Step 3 Model validation}

We will block coefficient $k_{j}$, average speed $v_{0}$ at the time of zero flow and mixed flow into the average speed $\bar{v}$ of these parameters (8), about 5, 90, 405, and 520 road traffic simulation calculation, obtained under different on the proportion of road traffic. The results are as shown in Table 4.

Table 4 shows that when the driving on the proportion between $10 \%-70 \%$, the stretch of road traffic is only the self-drive road traffic to reduce, and when the self-drive delivery ratio above $70 \%$, the stretch of road traffic significantly higher than that of only the self-drive road traffic. Results are basically consistent with the above theoretical analysis.

\subsection{Analysis of the Result}

In order to further validate our conclusion, the rationality of the velocity-flow model is established and calculated under different on the proportion of road traffic, as shown in Table 4, since the driving input to a certain extent, can actually increase the road capacity. When driving on the ratio between $10 \%-70 \%$, the stretch of road traffic is lower than only the self-drive road traffic, and when the self-drive delivery ratio above $70 \%$, the stretch of road traffic significantly higher than that of only the self-drive road traffic. It is also consistent with our vehicles in this paper the relationship between average speed ratio coefficient and mixing road.

\section{Conclusion}

Through the above analysis, we concluded that when the driving on the tipping 
Table 4. In each part of the road traffic simulation calculation results.

\begin{tabular}{|c|c|c|c|c|c|c|c|c|c|c|c|c|}
\hline Rou & Star & End & 0 & $10 \%$ & $20 \%$ & $30 \%$ & $40 \%$ & $50 \%$ & $60 \%$ & $70 \%$ & $80 \%$ & $90 \%$ \\
\hline 5 & 100.93 & 101.87 & 6500 & 64,441 & 63,592 & 60,492 & 59,967 & 60,198 & 61,016 & 65,392 & 69,439 & 74,435 \\
\hline 5 & 101.87 & 103.17 & 85,000 & 82,213 & 80,689 & 78,555 & 79,139 & 77,876 & 79,768 & 87,036 & 92,954 & 94,632 \\
\hline 5 & 103.17 & 103.42 & 108,000 & 107,718 & 104,230 & 102,251 & 100,918 & 98,426 & 102,491 & 109,671 & 115,172 & 121,168 \\
\hline 5 & 103.42 & 104.81 & 101,000 & 101,814 & 96,983 & 93,928 & 94,268 & 94,291 & 97,284 & 101,195 & 110,261 & 113,215 \\
\hline 5 & 104.81 & 105.63 & 144,000 & 144,111 & 139,951 & 134,188 & 132,234 & 131,349 & 138,550 & 146,979 & 156,448 & 163,125 \\
\hline 5 & 105.63 & 106.23 & 123,000 & 120,271 & 118,952 & 116,400 & 112,257 & 113,363 & 117,849 & 126,851 & 131,878 & 138,749 \\
\hline 5 & 106.23 & 107.09 & 143,000 & 141,044 & 136,960 & 134,101 & 131,554 & 129,897 & 136,787 & 144,993 & 154,294 & 161,905 \\
\hline 90 & 1.94 & 2.04 & 13,000 & 13,624 & 13,622 & 11,064 & 10,362 & 12,540 & 12,572 & 11,373 & 14,318 & 16,487 \\
\hline 90 & 2.04 & 2.4 & 44,000 & 42,141 & 44,142 & 43,456 & 40,198 & 40,108 & 41,304 & 43,530 & 47,197 & 51,600 \\
\hline 90 & 2.4 & 2.54 & 23,000 & 22,827 & 23,802 & 22,545 & 22,620 & 22,269 & 20,898 & 24,812 & 23,085 & 27,543 \\
\hline 90 & 2.54 & 2.79 & 23,000 & 23,665 & 21,662 & 20,407 & 20,162 & 21,818 & 20,486 & 22,255 & 26,051 & 25,167 \\
\hline 405 & 0 & 0.09 & 75,000 & 76,154 & 73,434 & 71,480 & 69,242 & 70,702 & 70,275 & 78,301 & 81,162 & 84,328 \\
\hline 405 & 0.09 & 0.37 & 110,000 & 107,338 & 107,047 & 104,788 & 100,101 & 99,640 & 104,950 & 110,705 & 117,876 & 123,697 \\
\hline 405 & 0.37 & 0.54 & 169,000 & 167,564 & 163,762 & 160,859 & 154,890 & 154,211 & 160,862 & 174,094 & 179,787 & 192,726 \\
\hline 405 & 0.54 & 0.75 & 176,000 & 173,921 & 170,119 & 166,163 & 164,215 & 162,871 & 170,889 & 179,841 & 189,466 & 200,169 \\
\hline 520 & 0 & 0.36 & 48,000 & 47,413 & 46,140 & 44,157 & 43,533 & 44,203 & 46,132 & 48,699 & 51,041 & 52,886 \\
\hline 520 & 0.36 & 0.85 & 79,000 & 78,255 & 75,589 & 73,549 & 74,231 & 72,697 & 77,210 & 81,160 & 83,491 & 88,078 \\
\hline 520 & 0.85 & 1.43 & 45,000 & 46,211 & 45,263 & 41,374 & 42,904 & 40,015 & 42,589 & 47,264 & 47,029 & 52,221 \\
\hline 520 & 1.43 & 1.63 & 61,000 & 59,239 & 57,717 & 58,379 & 54,919 & 57,788 & 58,873 & 61,342 & 63,852 & 70,598 \\
\hline 520 & 1.63 & 4.4 & 68,000 & 66,701 & 66,067 & 65,190 & 62,865 & 63,394 & 64,961 & 69,009 & 73,941 & 78,245 \\
\hline
\end{tabular}

point $k_{1}=38.63 \%$, it prevents traffic capacity of the largest. It influences the driving traffic capacity. When the delivery ratio $k<k_{1}$, self-drive for road traffic capacity has obvious inhibiting effect. When put in the proportion $k_{1}<k<k_{2}$, they have a positive influence to the traffic. When dropping ratio $k_{1}>k_{2}$, they have the significant improvement to the traffic capacity of the road. In this paper, we only consider the ordinary road traffic capacity. For certain capacity, we need to continue to look for a function of flow rate, density, time between.

\section{Acknowledgements}

The authors gratefully acknowledge the very helpful comments and suggestions of the editor and the anonymous reviewers for their valuable comments and suggestions that helped improve this paper.

\section{References}

[1] Lu, Z. (2008) Simulation Study on the Speed and Capacity of the Freeway Mixed Flows. Hunan University.

[2] Daganzo, C. and Knoop, V. (2016) Traffic Flow on Pedestrianized Streets. Transportation Research Part B: Methodological, 86, 211-222.

[3] Daganzo, C. and Lehe, L. (2016) Traffic Flow on Signalized Streets. Transportation Research Part B: Methodological, 90, 56-69.

[4] Soriguera, F., Martínez, I., Sala, M. and Menéndez, M. (2017) Effects of Low Speed 
Limits on Freeway Traffic Flow. Transportation Research Part C: Emerging Technologies, 77, 257-274.

[5] Jamshidnejad, A., Papamichail, I., Papageorgiou, M. and Schutter, B. (2017) A Mesoscopic Integrated Urban Traffic Flow-Emission Model. Transportation Research Part C: Emerging Technologies, 75, 45-83.

[6] Yang, K., Yang, L. and Gao, Z. (2016) Planning and Optimization of Intermodal Hub-and-Spoke Network under Mixed Uncertainty. Transportation Research Part E: Logistics and Transportation Review, 95, 248-266.

[7] Celikoglu, H. and Silgu, M. (2016) Extension of Traffic Flow Pattern Dynamic Classification by a Macroscopic Model Using Multivariate Clustering. Transportation Science, 50, 966-981. https://doi.org/10.1287/trsc.2015.0653

[8] Yang, K., Yang, L. and Gao, Z. (2017) Hub-and-Spoke Network Design Problem under Uncertainty Considering Financial and Service Issues: A Two-Phase Approach. Information Sciences, 402, 15-34.

[9] Chen, L., Peng, J. and Zhang, B. (2017) Uncertain Goal Programming Models for Bicriteria Solid Transportation Problem. Applied Soft Computing, 51, 49-59.

[10] Chen, L., Peng, J., Zhang, B. and Li, S. (2017) Uncertain Programming Model for Uncertain Minimum Weight Vertex Covering Problem. Journal of Intelligent Manufacturing, 28, 625-632. https://doi.org/10.1007/s10845-014-1009-1

[11] Gupta, A. and Dhiman, I. (2015) Phase Diagram of a Continuum Traffic Flow Model with a Static Bottleneck. Nonlinear Dynamics, 79, 663-671.

https://doi.org/10.1007/s11071-014-1693-6

[12] Qu, X., Wang, S. and Zhang, J. (2015) On the Fundamental Diagram for freeway Traffic: A Novel Calibration Approach for Single-regime Models. Transportation Research Part B: Methodological, 73, 91-102. https://doi.org/10.1016/j.trb.2015.01.001

[13] Tang, T., Chen, L., Wu, Y. and Caccetta, L. (2015) A Macro Traffic Flow Model Accounting for Real-Time Traffic State. Physica A: Statistical Mechanics and Its Applications, 437, 55-67. https://doi.org/10.1016/j.physa.2015.05.054

[14] Chen, L., Peng, J., Zhang, B. and Rosyida, I. (2017) Diversified Models for Portfolio Selection Based on Uncertain Semivariance. International Journal of Systems Science, 48, 637-648. https://doi.org/10.1080/00207721.2016.1206985

[15] Chen, L., Peng, J., Liu, Z. and Zhao, R. (2017) Pricing and Effort Decisions for a Supply Chain with Uncertain Information. International Journal of Production Research, 55, 264-284. https://doi.org/10.1080/00207543.2016.1204475

[16] Lan, B., Peng, J. and Chen, L. (2015) An Uncertain Programming Model for Competitive Logistics Distribution Center Location Problem. American Journal of Operations Research, 5, 536-547. https://doi.org/10.4236/ajor.2015.56042

[17] Liu, X., Peng, J. and Chen, L. (2015) Uncertain Programming Model for Location Problem of Multi-Product Logistics Distribution Centers. Applied Mathematical Sciences, 9, 6543-6558. https://doi.org/10.12988/ams.2015.59597

[18] Cheng, L., Rao, C., Chen, L. and Rosyida, I. (2016) A Belief Degree Constrained Programming Model for Maximum Cut Problem with Uncertain Edge Weights, ICIC Express Letters. Part B, Applications, 7, 1185-1191. 
Submit or recommend next manuscript to SCIRP and we will provide best service for you:

Accepting pre-submission inquiries through Email, Facebook, LinkedIn, Twitter, etc. A wide selection of journals (inclusive of 9 subjects, more than 200 journals)

Providing 24-hour high-quality service

User-friendly online submission system

Fair and swift peer-review system

Efficient typesetting and proofreading procedure

Display of the result of downloads and visits, as well as the number of cited articles Maximum dissemination of your research work

Submit your manuscript at: http://papersubmission.scirp.org/

Or contact ajcm@scirp.org 